\title{
Localised thermal analysis of a packaging film
}

\author{
D.M. Price ${ }^{\mathrm{a}, *}$, M. Reading ${ }^{\mathrm{a}}$, A. Hammiche ${ }^{\mathrm{b}}$, H.M. Pollock ${ }^{\mathrm{b}}$, M.G. Branch ${ }^{\mathrm{c}, 1}$ \\ a IPTME, Loughborough University, Loughborough, LE11 3TU, UK \\ ${ }^{\mathrm{b}}$ School of Physics and Chemistry, Lancaster University, Lancaster, LA1 4YB, UK \\ ${ }^{\mathrm{c} C}$ Courtaulds plc., 101 Lockhurst Lane, Coventry, CV6 5RS, UK
}

\begin{abstract}
A scanning probe microscope fitted with a fine platinum wire active heating element was used to examine the topography of a $300 \mu \mathrm{m}$ thick cross-section of a seven-layer polyolefin laminate designed for a food packaging application. Localised thermomechanical analysis was carried out by positioning the probe over selected regions and heating the wire whilst detecting the change in cantilever deflection using the microscope stage. Different grades of polyethylene and a poly(ethylenevinyl alcohol) barrier layer could be identified by softening behaviour at a resolution of $10 \mu \mathrm{m}$ or better - even in cases where there were no obvious changes in image contrast. (C) 1999 Elsevier Science B.V. All rights reserved.
\end{abstract}

Keywords: Localised thermomechanical analysis; Scanning probe microscopy; Packaging films; Polyethylene

\section{Introduction}

Polyethylene (PE), low density polyethylene (LDPE), medium density polyethylene (MDPE) and high density polyethylene (HDPE) are widely used in different packaging applications in the form of flexible films and laminates for bags and as semi-rigid and rigid containers as well as in pipe extrusion and injection moulding of different items [1]. The type of polyethylene used in films is well known to affect their properties; however, identification of the grade of polymer, particularly for commercial films, remains difficult. Bulk analytical techniques such as nuclear magnetic resonance spectroscopy (NMR), and differential scanning calorimetry (DSC) are only able to

\footnotetext{
*Corresponding author. Fax: +44-1509-223949; e-mail: d.m.price@lboro.ac.uk

${ }^{1}$ Present address: Unilever Research Port Sunlight Quarry Road East Bebington Wirral L63 3JW U.K.
}

give a specimen-averaged picture of the properties of the sample. More elaborate chromatographic techniques such as temperature rising elution fractionation (TREF) can, in favourable cases, separate the constituents of a multi-component film but afford no information regarding the original location of each fraction [2]. Infra-red microscopy is able to give more localised information of spectral properties, but (in this instance) it is not easy to distinguish between different grades of PE.

Recent developments in the field of scanning probe microscopy have combined the high spatial resolution of this technique with the characterisation capabilities of thermal analysis. Thus, for the first time, it is possible to measure the thermal response of a specimen in a localised region rather than on a macroscopic scale. The "micro-" equivalents of well-known techniques such as DSC, thermomechanical and dynamic mechanical measurements have been described by Reading et al. [3]; in this paper we will illustrate 
the utility of this approach to characterise a multilayer packaging film.

\section{Experimental}

A sample of a multi-layer packaging film was supplied by Courtaulds plc. The construction comprised a central gas and flavour barrier layer surrounded by HDPE, with an LDPE outer surface designed to accept printing ink. Thin $(20 \mu \mathrm{m}$ thick) slivers of material were carefully cut using a microtome and mounted on a metal stub so that a smooth cross-section ( $300 \mu \mathrm{m}$ wide) could be presented to the microscope for imaging and subsequent thermal analysis.

The instrument used for this work was a TA Instruments $\mu$ TA 2990 Micro-Thermal Analyser based on the TopoMetrix Explorer TMX2100 scanning probe microscope. At the heart of the system is a small resistively heated wire loop mounted on the end of the cantilever arm of the microscope head [4]. This is rastered across the sample to measure the topography of the surface in the usual force feedback mode. In addition to this, the probe can be held at a constant temperature and the thermal feedback signal used to construct an image of the surface based on spatial variations in the specimen's apparent thermal conduc- tivity. Once an image has been acquired, the probe can be moved to specific positions on the surface to carry out thermal measurements. In this work, we will concentrate on localised thermomechanical analysis; in this mode, the displacement of the tip is measured as it is heated in contact with the sample - as the temperature is raised a point is reached where the sample softens resulting in a downward deflection of the cantilever. At the end of the experiment, the probe is heated to the maximum scanned temperature and raised clear of the surface. Imaging of the sample postlocalised thermal analysis shows craters where the measurements were carried out. Because of the small thermal mass of the probe, high heating rates (typically $10^{\circ} \mathrm{C} \mathrm{s}^{-1}$ or more) can be used. The low thermal conductivity of polymer samples means that only a small region $\left(10 \mu \mathrm{m}^{3}\right)$ is examined.

\section{Results and discussion}

Fig. 1(a) shows a shaded topographic image of the main part of the sample - this is composed of HDPE. Localised thermomechanical measurements were carried out from $30^{\circ} \mathrm{C}$ to $210^{\circ} \mathrm{C}$ at a heating rate of $50^{\circ} \mathrm{C} \mathrm{s}^{-1}$ on a diagonal line running from top-left to bottom-right across the specimen. Fig. 1(b) shows the sample post-analysis - a line of small craters (approxi-

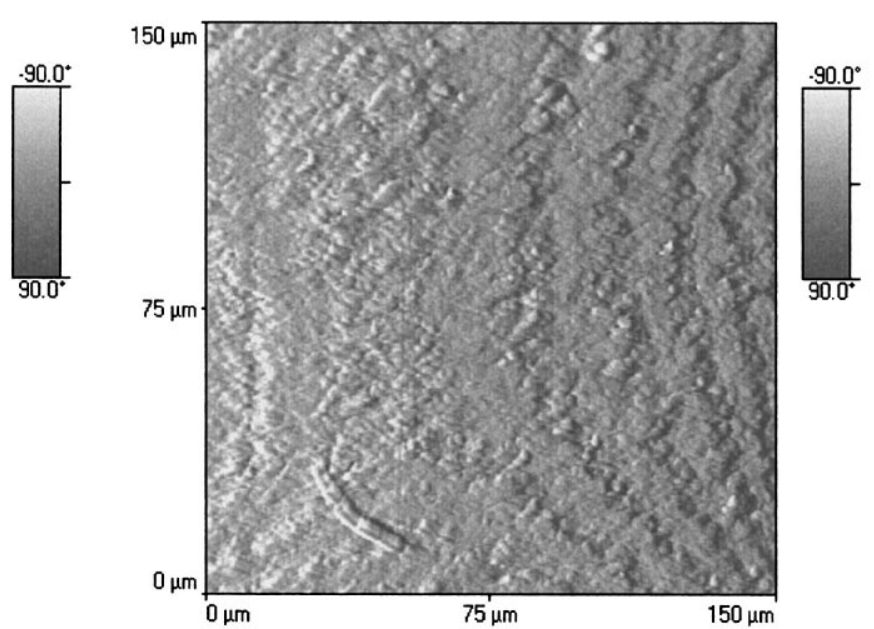

(a)

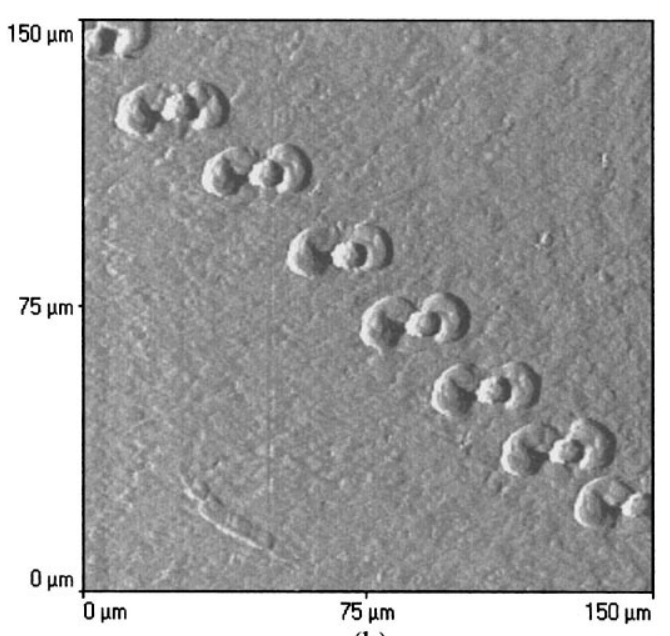

(b)

Fig. 1. Shaded topographic images of mid-section of multi-layer film comprising only HDPE: (a) before localised thermal analysis; (b) after localised thermal analysis. 


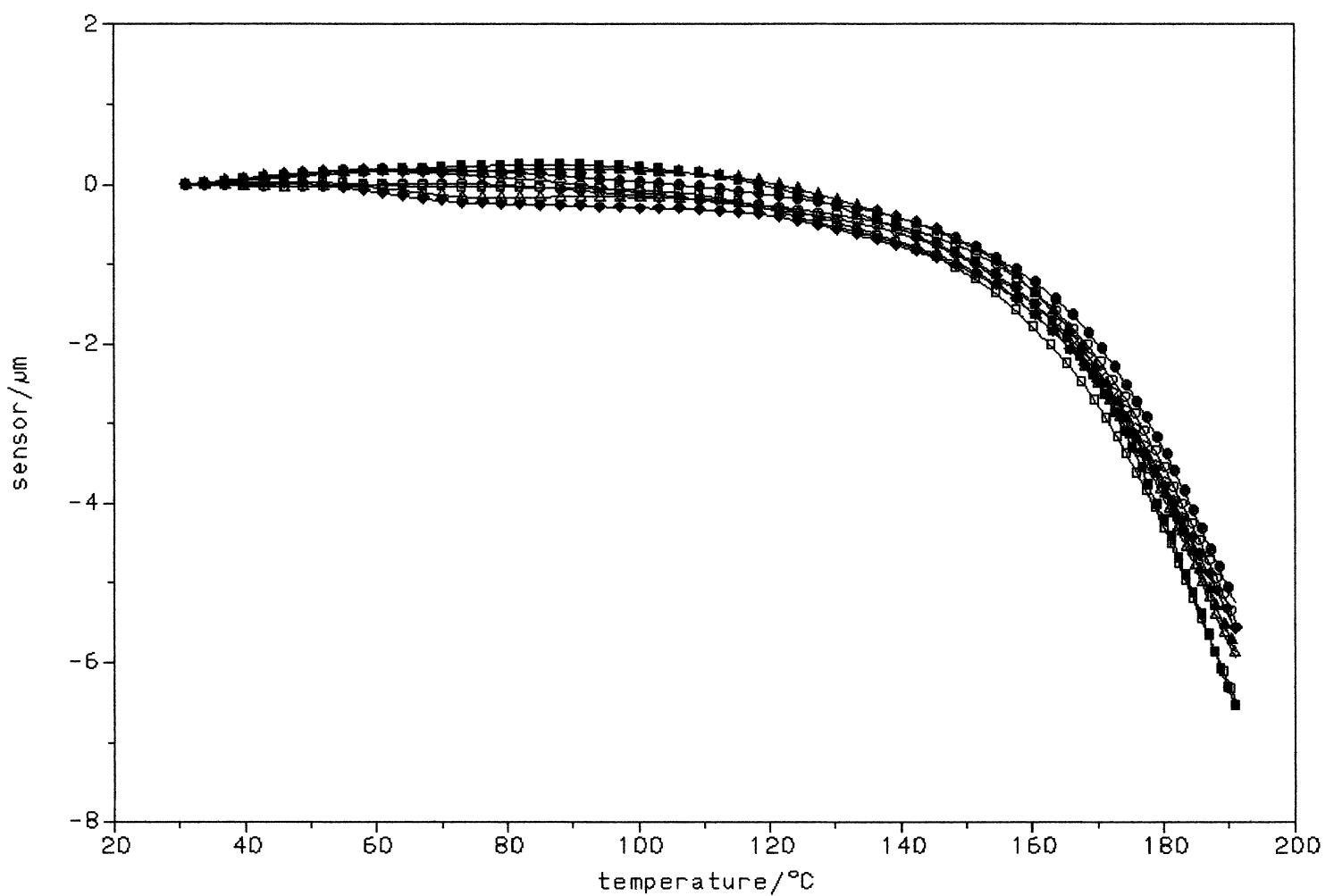

Fig. 2. Overlay of localised TMA curves from areas in Fig. 1(b) - sequence of scan locations $(\bigcirc: 1, \bullet: 2, \diamond: 3, \diamond: 4, \triangle: 5, \mathbf{\Delta}: 6, \square: 7$, 8) runs from top-left to bottom-right.

mately $20 \times 10 \mu \mathrm{m}^{2}$ ) can clearly be seen where the probe was pulled away from the sample following each test. The regions affected by the measurements appear large because the molten polymer flows away from the probe to cover the adjacent area. We estimate that the area characterised by the onset of probe penetration to be of the order of a few square microns. Plots of cantilever deflection (obtained from the photodetector feedback circuit in the microscope head) versus temperature for each location are shown in Fig. 2. The results show excellent reproducibility and the curves can be used to "fingerprint" the polymer type. Such a sequence of measurements can be conducted in a matter of a few minutes because of the high heating rates that are employed.

Fig. 3 shows a three-dimensional representation of the gas barrier layer in the packaging film - it is clear from the topography that there is a central layer of polymer flanked by two outer layers with possibly an intermediate tie layer. Localised thermomechanical measurements were carried out at six selected positions across the sample (Fig. 4). Plots of sensor response versus temperature are shown in Fig. 5; these correspond to the locations indicated above. The softening temperatures are indicative of the polymer present in the film: curves 1 and 5 are typical of HDPE, curves 3 and 6 correspond to the EVOH layer, curve 2 (the tie layer) has a lower softening point than HDPE and is probably MDPE, curve 4 is located on the $\mathrm{EVOH} /$ tie layer interface and the thermal response is a combination of both.

Topographic images of the outer print surface preand post-localised thermomechanical measurements are shown in Fig. 6(a) and (b), respectively. The outside edge of the specimen is located in the top-right corner of each image. It is not clear from Fig. 6(a) where the boundary between the LDPE and HDPE occurs. A series of measurements were therefore carried out in a zigzag pattern starting from the edge of the sample and finishing in the centre of the image 


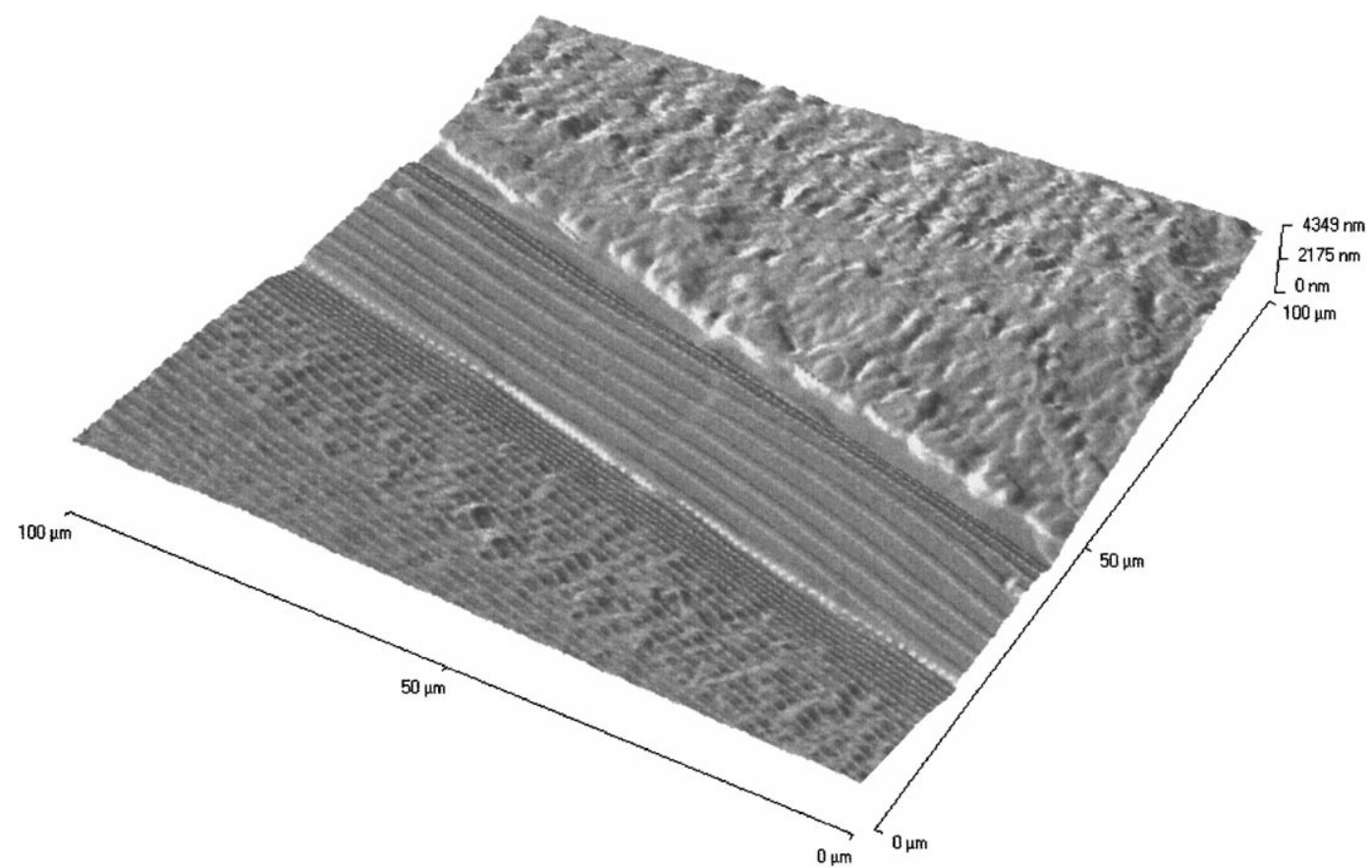

Fig. 3. Three-dimensional perspective representation of barrier layer region of film before localised thermal analysis.

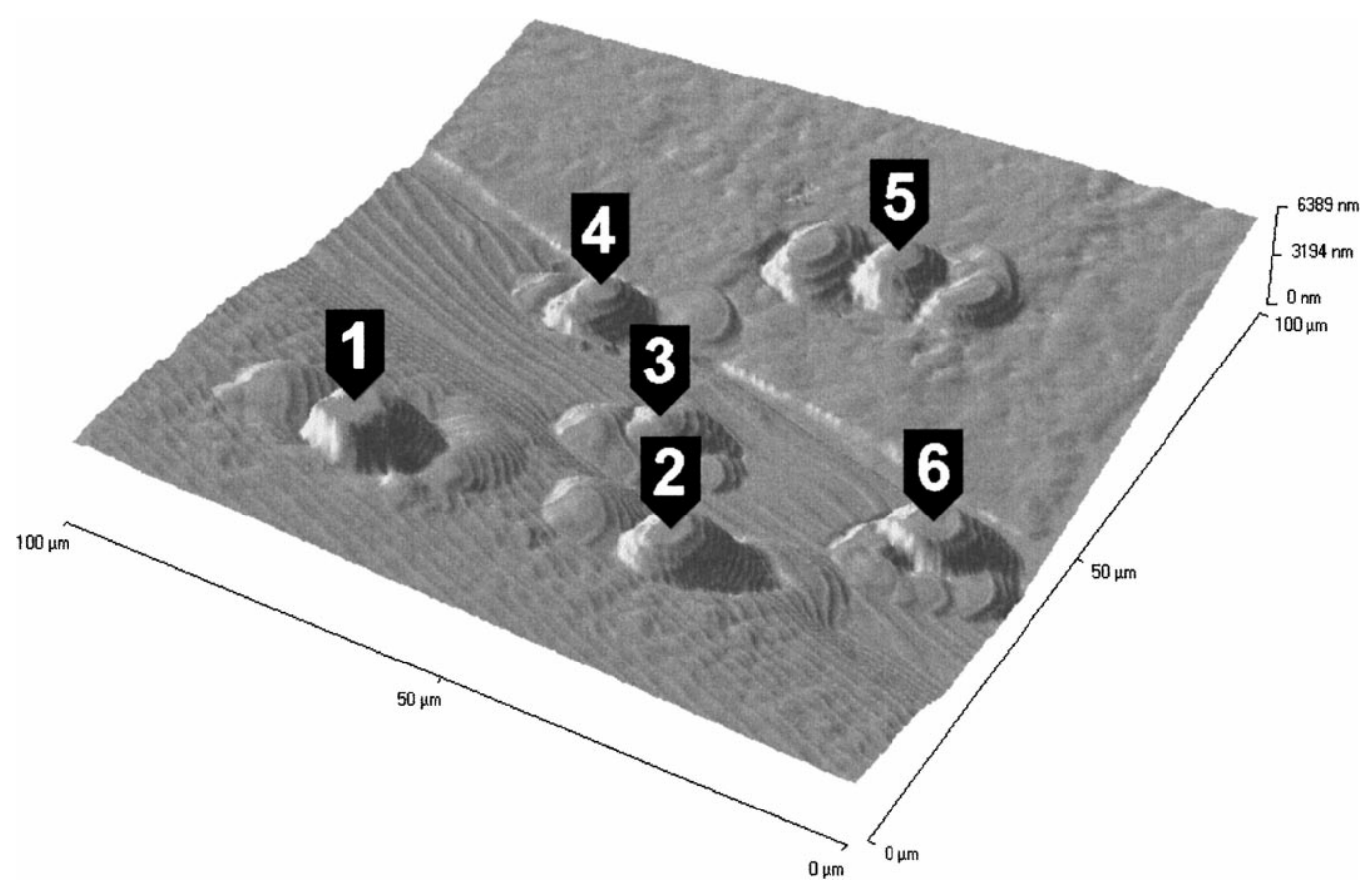

Fig. 4. Three-dimensional perspective representation of barrier layer region of film after localised thermal analysis - labels refer to sequence of scans. 


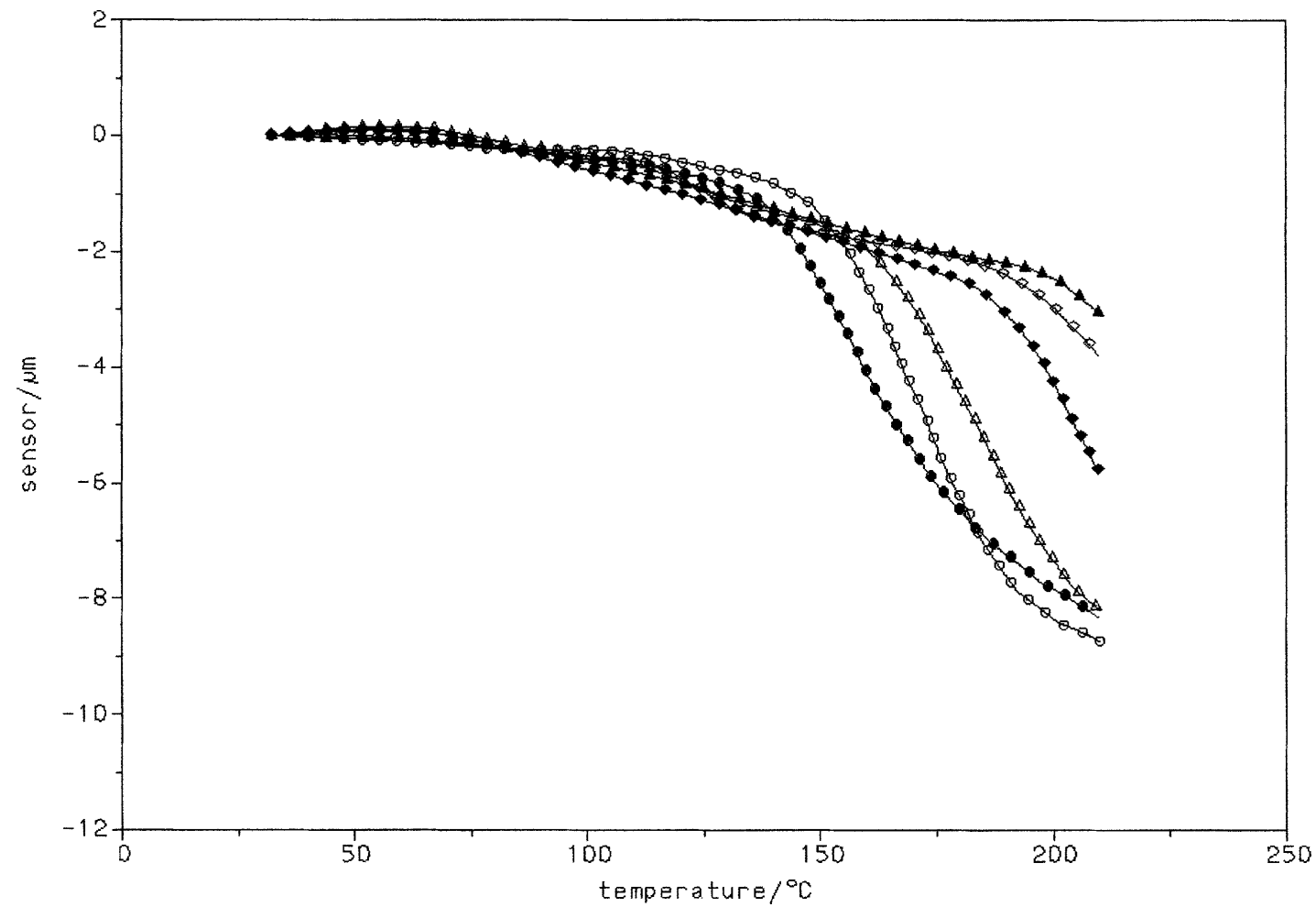

Fig. 5. Overlay of localised TMA curves from barrier layer region - for sequence of scan locations $(\bigcirc: 1, \bullet: 2, \diamond: 3, \diamond: 4, \triangle: 5, \mathbf{\Delta}: 6)$ see Fig. 4.

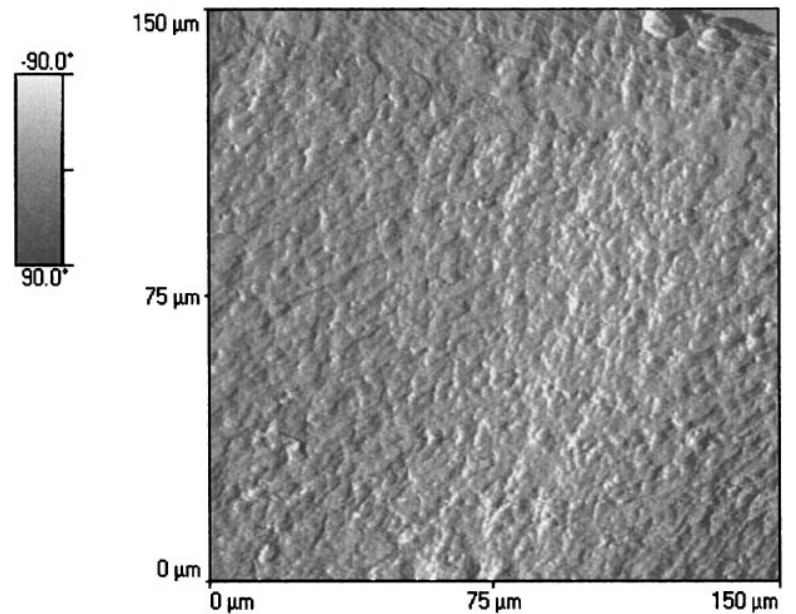

(a)

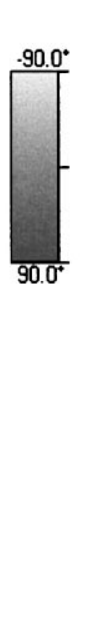

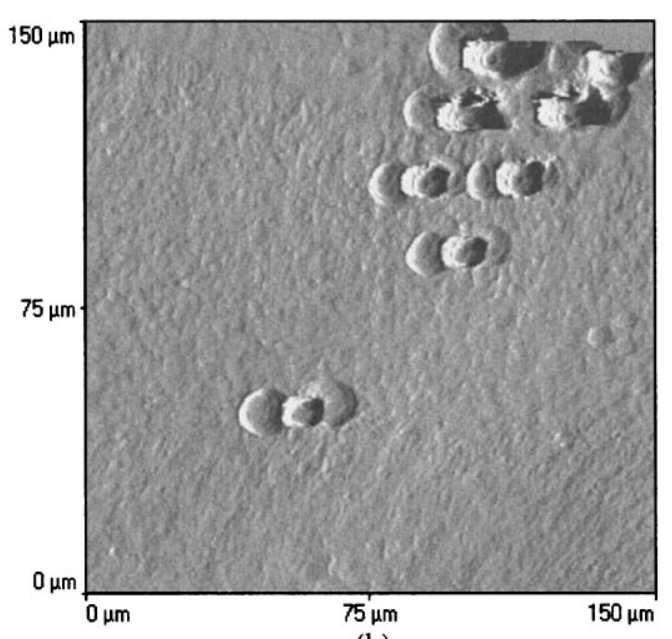

(b)

Fig. 6. Shaded topographic images of outer print surface of laminate: (a) before measurement; (b) after measurement. See text for sequence of scans. 


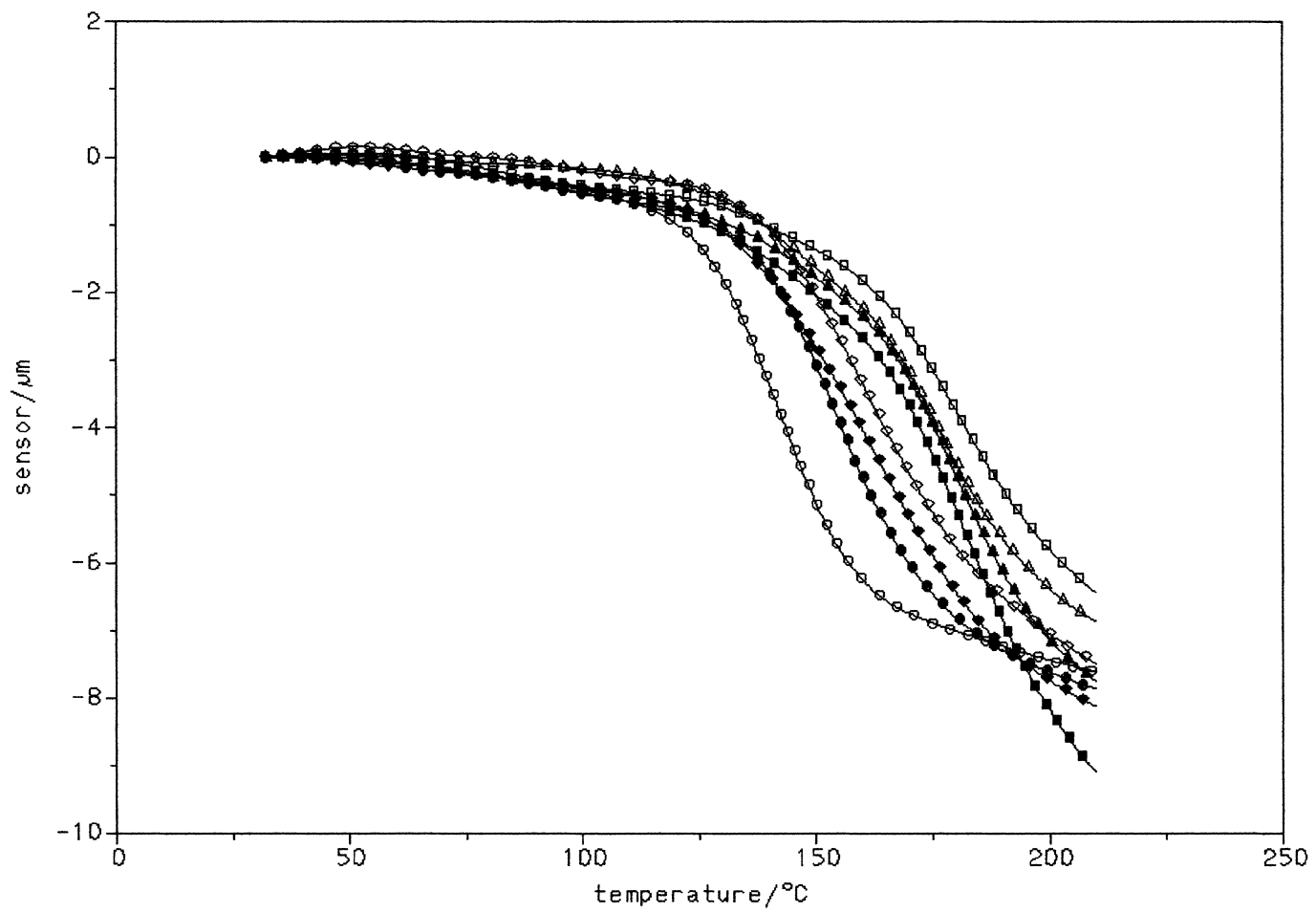

Fig. 7. Overlay of localised TMA curves from outer surface of laminate. Order of scan locations $(\bigcirc: 1$,

: 8) in increasing distance from outside edge of sample.

(Fig. 6(b)). The resulting thermal curves are shown in Fig. 7 - the sequence of tests is in increasing distance from the outside edge of the film. There is an increase in softening temperature from the first to the last test with an apparent change in response between scan locations 4 and 5, approximately $30-40 \mu \mathrm{m}$ from the print surface. Closer inspection of Fig. 6(a) reveals a subtle line running diagonally across the image from near the top-left to about two-thirds of the way up the right-hand side of the image. This feature probably represents the interface between the LDPE and HDPE layers.

\section{Conclusions}

The combination of topographic information obtained via scanning probe microscopy and the ability to perform localised thermal analysis with good spatial resolution affords a rapid method for investigating the composition of complex structures. In this study, different components in a multi-layer packaging film were identified from their thermomechanical properties. It is also possible to carry out localised modulated-temperature calorimetry which offers the opportunity to make qualitative measurements of differences in crystallinity [5]. By placing an appropriate transfer line to a mass spectrometer close to the heated wire loop, chemical identification of the evolved gases produced by using the probe to pyrolyse selected points could be envisaged thus completing the repertoire of micro-thermal analysis techniques.

\section{Acknowledgements}

The authors would like to thank Mr. E.J. Watson for help in specimen preparation.

\section{References}

[1] B. Wolf, S. Keng, J. Klopstock, J. Miltz, J. Appl. Polym. Sci. 62 (1996) 1339. 
[2] M. Keeting, I. Lee, C.S. Wong, Thermochim. Acta 284 (1996) 47.

[3] M. Reading, D.J. Hourston, M. Song, H.M. Pollock, A. Hammiche, Am. Lab. 30(1) (1998) 13.

[4] R.B. Dinwiddie, R.J. Pylkki, P.E. West, in: T.W. Tong (Ed.),
Thermal Conductivity, vol. 22, Technomics, Lancaster, PA, 1994, pp. 668-677.

[5] H.M. Pollock, A. Hammiche, M. Song, D.J. Hourston, M. Reading, J. Adhesion 67 (1998) 193. 